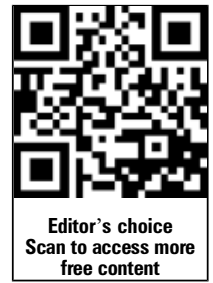

${ }^{1}$ Environmental Epidemiology Division, Institute for Risk Assessment Sciences, Utrecht University, Utrecht, Utrecht, The Netherlands

${ }^{2}$ Department of Epidemiology, GROW School for Oncology and Developmental Biology, Maastricht University MedicalCentre, Maastricht, The Netherlands

${ }^{3}$ Netherlands Organization for Applied Research (TNO), Leiden, The Netherlands ${ }^{4}$ Julius Centre for Public Health Sciences and Primary Care, University Medical Centre, Utrecht, The Netherlands

\section{Correspondence to} Dr Roel Vermeulen, Division of Environmental Epidemiology, Institute for Risk Assessment Sciences, Utrecht University, P.O. Box 80.178, Utrecht 3584 TD, The Netherlands: r.c.h.vermeulen@uu.nl

Received 1 May 2012 Revised 30 November 2012 Accepted 15 December 2012 Published Online First 15 January 2013

\title{
Occupational exposure to extremely low-frequency magnetic fields and cardiovascular disease mortality in a prospective cohort study
}

\author{
Tom Koeman, ${ }^{1}$ Pauline Slottje, ${ }^{1}$ Hans Kromhout, ${ }^{1}$ Leo J Schouten, ${ }^{2}$ \\ R Alexandra Goldbohm, ${ }^{3}$ Piet A van den Brandt, ${ }^{2}$ Roel Vermeulen ${ }^{1,4}$
}

\begin{abstract}
Objectives Although a study among utility workers found an increased risk for acute myocardial infarction and arrhythmia-related deaths associated with occupational extremely low-frequency magnetic fields (ELF-MF) exposure, later studies largely failed to replicate these findings. This study investigated the association between occupational ELF-MF exposure and cardiovascular disease (CVD) mortality within a community-based prospective cohort study.

Methods The Netherlands Cohort Study is a prospective cohort study among 120852 men and women aged 55-69 years at baseline. Participants were followed-up for CVD mortality over a period of 10 years, resulting in 8200 CVD deaths. Information on occupational history and potential confounders, such as educational level, smoking and alcohol use were collected at baseline through a self-administered questionnaire. Occupational ELF-MF exposure was assigned using a job-exposure matrix. Associations with CVD mortality were analysed using Cox regression. Results Ever low or high exposure to ELF-MF showed no association with total CVD mortality (HR of 1.02, $95 \% \mathrm{Cl} 0.99$ to 1.06$)$, nor with any cause-specific subtypes of CVD mortality. Other ELF-MF exposure metrics showed no increased risks either.

Conclusions In this study, we found no indication of

\section{What this paper adds}

- A large industrial cohort study amongst electrical utility workers has shown an association between occupational exposure to extremely low-frequency magnetic fields (ELF-MF) and increased mortality due to acute myocardial infarctions and arrhythmias.

- Other studies have failed to replicate this finding, but subsequent occupational studies suffered from low numbers of cases, while subsequent general-population studies were limited to incomplete job histories.

- This study, using The Netherlands Cohort Study, improves upon earlier replication efforts by using a large, prospective, general-population cohort with a large number of cases, and with complete job histories up to enrolment.

- This study did not find an association between occupational exposure to ELF-MF and mortality due to (subtypes of) cardiovascular disease (CVD), adding to the combined evidence that exposure to ELF-MF does not increase the risk of death from CVD.
\end{abstract} an association between occupational ELF-MF exposure and risk of CVD mortality.

\section{INTRODUCTION}

The possible health effects of exposure to extremely low-frequency magnetic fields (ELF-MF) have been studied for three decades. ${ }^{1}$ ELF-MF are magnetic fields produced by electrical appliances and the power grid, with a frequency up to $300 \mathrm{~Hz}$. Experimental evidence in humans pointing to a possible effect of ELF-MF on heart rate variability (HRV) ${ }^{2}{ }^{3}$ and, ultimately, on acute cardiac diseases, led Savitz et $a l^{4}$ to investigate the effects of occupational exposure to ELF-MF on cardiovascular disease (CVD) mortality in a cohort of utility workers. Their findings suggested a positive association between occupational ELF-MF exposure and mortality due to cardiac arrhythmia and acute myocardial infarction (AMI). Although some studies have offered marginal support for this early observation, ${ }^{5}$ others have failed to replicate these findings. ${ }^{6-11}$ A recent review, therefore, concluded that the current evidence does not support a relationship between occupational exposures to ELF-MF and CVD mortality. ${ }^{12}$

The majority of the epidemiological studies to date have been performed in industry-based populations. ${ }^{4} 591113$ While industry-based studies offer the prospect of a detailed occupational exposure assessment, they often have limited ability to adjust for potential confounding factors, such as smoking and alcohol use. In addition to the industry-based studies, three general population-based cohort studies on ELF-MF and CVD-related mortality have been published. ${ }^{8} 10$ These may have the advantage of both complete occupational histories which minimises potential healthy worker inclusion effects, and detailed information on possible confounders, albeit that this often comes at the cost of less detailed occupational exposure assessment, as well as a lower prevalence of high exposures. The aforementioned general population studies on ELF-MF suffered, however, from one or more limitations of either being limited to information on a single occupation instead of the full occupational 
history, ${ }^{8} 10$ or because information on potentially important confounders was missing and, instead, estimated indirectly. ${ }^{7}$

In this study, we addressed these limitations by investigating the association between occupational ELF-MF exposure and CVD mortality using a large community-based prospective cohort study ${ }^{14}$ with complete occupational history and detailed confounder information up to baseline.

\section{MATERIALS AND METHODS Population}

The Netherlands Cohort Study on diet and cancer (NLCS) consists of 120852 subjects (58 279 men and 62573 women) who were enrolled in 1986. Subjects were aged 55-69 years and living in 204 municipalities throughout The Netherlands at the time of enrolment. ${ }^{14}$ Participants completed a questionnaire at baseline on occupational history, dietary habits and other potential risk factors for cancer. The first page, containing basic demographic information, was machine readable and entered for all subjects. Following a case-cohort approach, the remaining pages were entered manually for a randomly drawn subcohort $(n=5000)$. After enrolment, the whole cohort was followed-up for cause-specific mortality and cancer incidence. The remaining pages of the questionnaire were entered for incident cases.

The NLCS was approved by the institutional review boards of the Netherlands Organization for Applied Scientific Research TNO (Zeist) and Maastricht University (Maastricht).

\section{Case definition}

Deaths were hand searched from the Dutch Bureau of Genealogy for the period from January 1987 through to October 1994, and obtained through record linkage for the remaining period up to December 1996, resulting in a 10 -year follow-up period. Causes of deaths were obtained from the Central Bureau of Statistics (CBS) and coded according to the 9th, and from 1 January 1996 onwards, the 10th revision of the International Classification of Diseases. For this analysis, causes of death were grouped into total CVD mortality, ischaemic heart disease (IHD), AMI, subacute and chronic IHDs, other cardiac diseases, arrhythmias, atherosclerosis and cerebrovascular disease mortality (see table 1). In total, 8200 CVD deaths, either as primary or secondary causes of death, were traced in the follow-up of the cohort. Of these, information on occupational history and possible confounders was available for 6151 cases.

Table 1 Case definition and number of cases of cardiovascular mortality

\begin{tabular}{llll}
\hline Disease outcome & ICD-9 coding & ICD-10 coding Number of cases \\
\hline Total cardiovascular mortality & $390-459$ & $100-199$ & 6151 \\
IHD & $410-414$ & $120-125$ & 3292 \\
AMI & 410 & 121,122 & 2281 \\
Subacute and chronic IHDs & $411-414$ & $120,124,125$ & 1015 \\
Other cardiac diseases & $420-429$ & $130-152$ & 1084 \\
Arrhytmias & $426-427$ & 144,145, & 147 \\
& excluding & $147-149$ & \\
Atherosclerosis-related & $427.5 *$ & & 121 \\
mortality & 440 & I70 & \\
Cerebrovascular diseases & $430-438$ & I60-I69 & 1143 \\
\hline *Cardiac arrest (ICD9: 427.5$)$ & is excluded from the definition of arrhythmias. \\
AMI, acute myocardial infarction; ICD, International Classification of Diseases; IHD, \\
Ischaemic heart disease.
\end{tabular}

\section{Assignment of occupational ELF-MF exposure}

The questionnaire included questions on the occupational history of the participants up to baseline. Participants were asked to report job title, company name and type of industry, products manufactured and time period for each job they ever performed. Of the cohort, $78 \%$ of them were not employed at baseline. Participants could enter up to five jobs in the questionnaire. Participants in the subcohort entered, on average, 1.9 jobs. This information was coded into job codes by a dedicated research assistant according to the Standard Occupational Classification of the Dutch CBS from 1984 (CBS-84). ${ }^{15}$ These CBS-84 job codes were translated into ISCO-88 using an automated cross-walk developed for this purpose. ${ }^{16}$

Subsequently, exposure to ELF-MF was assigned to each job by linking the ISCO-88 job codes to a recently developed ELF-MF job-exposure matrix (JEM). ${ }^{17}$ This JEM is a modified version of a previously published ELF-MF JEM developed by Bowman et al. ${ }^{18}$ The original ELF-MF JEM reflects the intensity of timeweighted average exposure in micro-Tesla $(\mu \mathrm{T})$ by job based on available measurement data, but does not account for the probability of exposure. To account for both the intensity and probability of exposure, geometric mean intensities were first categorised into background (i.e. very low), low and high ELF-MF exposure levels based on distributional cut points at $0.15 \mu \mathrm{T}$ and $0.30 \mu \mathrm{T}$. The resulting intensity-based ratings (background, low, high) were subsequently up- or downgraded by two industrial hygienists (HK and RV) based on the estimated probability of exposure per job, and classified as background low or high exposure. Median intensity of these categories based on the original JEM were $0.11,0.19$ and $0.52 \mu \mathrm{T}$, respectively.

Unpaid jobs were assigned the background exposure level. If job descriptions were missing or unable to be coded $(12 \%$ of cases and $13 \%$ of subcohort), exposure for that particular job was set to missing. Missing job durations were imputed based on sex-specific average job durations in the cohort $5 \%$ of cases and $4 \%$ of subcohort). Some subjects filled out overlapping job periods. In these cases, the overlapping years were divided equally over the overlapping jobs.

The following exposure metrics were considered:

- Ever 'low or high exposure' versus 'background exposure' to ELF-MF: the ever low and high categories were merged because the small numbers of high exposed subjects in the subcohort led to unstable risk estimates;

- Exposure duration: the period of time (in years) a subject reported to have performed a job with low or high ELF-MF exposure up to baseline;

- Cumulative exposure: level of ELF-MF exposure over the job history up to baseline. This was calculated by first multiplying, per job, the duration with exposure level using weights based on the distribution of the intensity of ELF-MF exposure over the occupations in the original ELF-JEM (0 for background, 1 for low and 4 for high exposure). The resulting unit-years per job were then summed across all jobs in the job history. Cumulative exposure was analysed as a categorical variable, using background exposure (cumulative exposure of 0 ) as the reference category and dividing those with cumulative exposures higher than background in three categories based on the tertiles of the exposed in the subcohort. In addition, a test for linear trend was performed by entering the cumulative exposure as a continuous variable, both among all subjects and among those with a low or high exposure to ELF-MF. 


\section{Statistical analysis}

In this case-cohort study, the association between the different exposure metrics of ELF-MF and CVD mortality was tested with Cox proportional hazards models, using the stcox procedure of STATA V.10 (Statacorp LP, College Station, Texas, USA). Person-time estimates were derived from the randomly sampled subcohort. CIs (95\%) were calculated using the 'robust' option. Attained age was used as the underlying time scale in the Cox models.

Models were corrected for sex, age at baseline (5 year-categories), current smoking status (yes or no), average number of cigarettes smoked per day, number of years of cigarette smoking, alcohol use (grams per day), educational level attained (primary, lower, secondary and medium, and university and higher vocational) and Body Mass Index. Other potential confounders were also tested, that is, marital status (never married, married, divorced and widower), smoking status of the partner (non-, former and current smoker), ever exposed to cigarette smoke in the workplace (never, sometimes, often, always), physical activity outside work (continuous (minutes per day) and categorical (less than $30 \mathrm{~min} /$ day, $30-60 \mathrm{~min} /$ day, 60-90 $\mathrm{min} /$ day and more than $90 \mathrm{~min} /$ day)), consumption of fruit, legumes, vegetables, meat products and fish products (all in average grams per day) and total energy intake (kilo calories per day). However, these confounders were not included in the final models as they did not affect the point estimates, yet reduced the number of cases included due to missing data. Analyses were performed on the entire cohort, as well as stratified by sex.

\section{RESULTS}

\section{Population}

Subjects with assigned exposure levels and complete confounder information were included in the analyses. This entailed 6151 cases $(75 \%$ of total number of cases) and a subcohort of $\mathrm{n}=3881$ (78\% of complete subcohort) in the analysis of total CVD mortality. The number of cases included in the analyses of the subtypes of CVD disease mortality are shown in table 1 . Subjects included in the analyses were similar for most (potential) confounders compared with excluded subjects, although the latter more often had a lower level of attained education.
Approximately half the cases and the subcohort members had ever been low or high exposed to ELF-MF over their entire job history. Of these, approximately $7 \%$ had ever been high exposed $(8.1 \%$ of the men compared with $0.6 \%$ of the women in the subcohort). The proportion of subjects with ever high exposure was higher among all cases (7.7\%) compared with the subcohort (4.4\%) (see table 2). Men had longer working histories than women (on average, 36 vs 13 years). Of these working years, men and women worked on average 14 and 6 years, respectively, in jobs with low or high ELF-MF exposure. Because of the higher exposure levels as well as the longer job history, men were more likely to be in the third tertile of cumulative ELF-MF exposure, while women were more likely to fall in the first tertile.

\section{Occupational ELF-MF exposure and risk factors for cardiovascular mortality}

Subjects with a higher cumulative ELF-MF exposure showed some differences in potential risk factors for CVD as compared with subjects with a lower cumulative exposure (see table 3 ). Subjects within the highest tertile of cumulative exposure were more likely to be current or former smokers and, on average, smoked more cigarettes per day, and smoked for a longer period of time. Subjects within the highest tertile of cumulative exposure also were more likely to drink larger amounts of alcohol per day. This difference was explained to a large extent by differences in the sex distribution between subjects with a lower or higher cumulative exposure. When stratified by sex, no significant differences remained.

\section{Occupational ELF-MF exposure and cardiovascular mortality}

No statistically significant association was detected between occupational ELF-MF exposure and total CVD mortality for any of the exposure metrics in the main adjusted analyses (HR for ever low or high exposure versus background exposure to ELF-MF was $1.02,95 \%$ CI 0.99 to 1.06 , see table 4 ) as well as in the sex-stratified analyses (HR for men 1.02, 95\% CI 0.98 to 1.06 , and for women HR 1.00, 95\% CI 0.93 to 1.07). Additional analyses excluding those with self-reported

Table 2 Age category, smoking status, numbers of cigarettes smoked per day and number of years smoked, alcohol use, educational level, body mass index and occupational exposure to extremely low-frequency magnetic fields for the subcohort and all cardiovascular deaths (cases) by sex

\begin{tabular}{|c|c|c|c|c|c|c|c|c|c|c|c|c|}
\hline & \multicolumn{6}{|c|}{ Subcohort } & \multicolumn{6}{|c|}{$\begin{array}{l}\text { Cases } \\
\text { Total cardiovascular mortality }\end{array}$} \\
\hline & \multicolumn{3}{|l|}{ Male } & \multicolumn{3}{|c|}{ Female } & \multicolumn{3}{|l|}{ Male } & \multicolumn{3}{|c|}{ Female } \\
\hline & n & $\% /$ mean & SD & n & $\% / m e a n$ & SD & $\mathbf{n}$ & $\% / m e a n$ & SD & $\mathbf{n}$ & $\% /$ mean & SD \\
\hline \multicolumn{13}{|l|}{ Ever exposed to ELF-MF } \\
\hline Background (\%) & 926 & 48.8 & & 977 & 50.3 & & 2106 & 46.8 & & 825 & 49.9 & \\
\hline Low (\%) & 855 & 44.1 & & 954 & 49.1 & & 1934 & 43.0 & & 810 & 48.9 & \\
\hline High (\%) & 156 & 8.1 & & 13 & 0.6 & & 456 & 10.1 & & 20 & 1.2 & \\
\hline Duration of ever low or high exposed (years; mean,SD) & 1011 & 26.7 & 14.2 & 967 & 11.2 & 9.5 & 2390 & 26.6 & 14.0 & 830 & 10.7 & 9.7 \\
\hline \multicolumn{13}{|l|}{ Cumulative exposure* Categorical } \\
\hline Background (\%) 0 unit-years & 926 & 47.8 & & 977 & 50.3 & & 2106 & 46.8 & & 825 & 49.8 & \\
\hline 1st tertile of exposed (\%) 0.5-9 unit-years & 160 & 8.3 & & 544 & 28.0 & & 348 & 7.7 & & 491 & 29.7 & \\
\hline 2nd tertile of exposed (\%) 9.5-28 unit-years & 272 & 14.0 & & 353 & 18.1 & & 682 & 15.2 & & 277 & 16.7 & \\
\hline 3rd tertileof exposed (\%) 28.5-208 unit-years & 579 & 29.9 & & 70 & 3.6 & & 1360 & 30.3 & & 62 & 3.8 & \\
\hline Continuous (unitxyears; mean, SD) & 1937 & 18.6 & 29.1 & 1944 & 5.7 & 9.4 & 4496 & 20.2 & 32.1 & 1655 & 5.6 & 9.2 \\
\hline
\end{tabular}


Table 3 Potential confounders by cumulative extremely low-frequency magnetic field exposure in the subcohort

\begin{tabular}{|c|c|c|c|c|c|c|c|c|c|c|c|c|}
\hline & \multicolumn{12}{|c|}{ Extremely low-frequency ELF } \\
\hline & \multicolumn{3}{|c|}{0 unit-years } & \multicolumn{3}{|c|}{$0.5-9$ unit-years } & \multicolumn{3}{|c|}{ 9.5-28 unit-years } & \multicolumn{3}{|c|}{ 28.5-208 unit-years } \\
\hline & n & $\% /$ mean & SD & n & $\% /$ mean & SD & n & $\% /$ mean & SD & n & $\% /$ mean & SD \\
\hline \multicolumn{13}{|l|}{ Sex $(\%)$} \\
\hline Male & 926 & 48.7 & & 143 & 23.6 & & 270 & 38.7 & & 598 & 88.6 & \\
\hline Female & 977 & 51.3 & & 463 & 76.4 & & 427 & 61.3 & & 77 & 11.4 & \\
\hline \multicolumn{13}{|l|}{ Age at baseline (\%) } \\
\hline $55-60$ years & 692 & 36.4 & & 251 & 41.4 & & 259 & 37.2 & & 133 & 29.8 & \\
\hline $60-65$ years & 656 & 34.5 & & 206 & 34.0 & & 237 & 34.0 & & 152 & 34.1 & \\
\hline $65-70$ years & 555 & 29.2 & & 149 & 24.6 & & 201 & 28.8 & & 161 & 36.1 & \\
\hline Age at baseline (mean, SD) Years & 1903 & 61.6 & 4.3 & 606 & 61.2 & 4.1 & 697 & 61.5 & 4.3 & 675 & 61.6 & 4.2 \\
\hline \multicolumn{13}{|l|}{ Smoked cigarettes (\%) } \\
\hline Never & 733 & 38.5 & & 288 & 47.5 & & 273 & 39.2 & & 100 & 14.8 & \\
\hline Former smoker & 644 & 33.8 & & 175 & 28.9 & & 243 & 34.9 & & 355 & 52.6 & \\
\hline Current smoker & 526 & 27.6 & & 143 & 23.6 & & 181 & 26.0 & & 220 & 32.6 & \\
\hline Number of cigarettes smoked per day (mean, SD) & 1903 & 9.6 & 11.2 & 606 & 7.5 & 10.9 & 697 & 9.2 & 10.8 & 675 & 13.5 & 10.3 \\
\hline Number of years smoking cigarettes (mean, SD) & 1903 & 19.5 & 18.3 & 606 & 15.4 & 17.5 & 697 & 18.8 & 17.8 & 675 & 28.4 & 15.9 \\
\hline \multicolumn{13}{|l|}{ Highest level of education attained (\%) } \\
\hline Primary vocational & 497 & 26.1 & & 186 & 30.7 & & 215 & 30.9 & & 155 & 23.0 & \\
\hline Lower vocational & 354 & 18.6 & & 148 & 24.4 & & 154 & 22.1 & & 185 & 27.4 & \\
\hline Secondary and medium vocational & 645 & 33.9 & & 233 & 38.5 & & 278 & 39.9 & & 259 & 38.4 & \\
\hline University and higher vocational & 407 & 21.4 & & 39 & 6.4 & & 50 & 7.2 & & 76 & 11.3 & \\
\hline Body Mass Index (mean, SD) $\mathrm{kg} / \mathrm{m}^{2}$ & 1903 & 24.9 & 3.1 & 606 & 25.3 & 3.4 & 697 & 25.2 & 3.2 & 675 & 24.9 & 2.6 \\
\hline Alcohol consumption (mean, SD) g/day & 1903 & 11.2 & 15.3 & 606 & 7.7 & 11.8 & 697 & 9.6 & 14.0 & 675 & 11.9 & 13.6 \\
\hline
\end{tabular}

cardiovascular conditions (hypertension, heart attack, angina pectoris, thrombosis and stoke) at baseline did not change these results.

Table 5 shows that there were no statistically significant associations between cumulative occupational ELF-MF exposure and the various subtypes of CVD mortality. Only the results for the most comprehensive metric (cumulative exposure) are shown as results for the other exposure metrics (ever low or high exposed vs background exposure and duration of low or high exposure) resulted in similar observations. Furthermore, stratified analyses by sex, smoking status and alcohol intake did not result in other insights.

We performed several sensitivity analyses to investigate the possible influence of alternative definitions of cases and of exposure. To test differing definitions of cases, we repeated the analyses of CVD and subtypes using only the primary cause of death. Using primary cause of death as the case definition did not materially change the results for CVD (third tertile of exposed vs background: HR $1.01,95 \%$ CI 0.88 to 1.15 ) or any

Table 4 Total cardiovascular disease mortality in relation to occupational extremely low-frequency magnetic field exposure

\begin{tabular}{|c|c|c|c|c|c|c|c|c|c|}
\hline & \multicolumn{3}{|l|}{ Total } & \multicolumn{3}{|l|}{ Men } & \multicolumn{3}{|c|}{ Women } \\
\hline & Cases & $\begin{array}{l}\text { Person-years in } \\
\text { subcohort }\end{array}$ & $\begin{array}{l}\text { Adjusted HR* } \\
(95 \% \mathrm{Cl})\end{array}$ & Cases & $\begin{array}{l}\text { Person-years in } \\
\text { subcohort }\end{array}$ & $\begin{array}{l}\text { Adjusted HR* } \\
(95 \% \mathrm{Cl})\end{array}$ & Cases & $\begin{array}{l}\text { Person-years in } \\
\text { subcohort }\end{array}$ & $\begin{array}{l}\text { Adjusted HR* } \\
(95 \% \mathrm{Cl})\end{array}$ \\
\hline \multicolumn{10}{|l|}{ Ever exposed } \\
\hline Background & 2931 & 17726 & 1 & 2106 & 8434 & 1 & 825 & 9293 & 1 \\
\hline Low/high & 3220 & 18461 & 1.02 (0.93 to 1.13$)$ & 2390 & 9214 & 1.03 (0.91 to 1.16$)$ & 830 & 9247 & 0.96 (0.83 to 1.12$)$ \\
\hline $\begin{array}{l}\text { Exposure duration } \\
\text { (low or high exposed) } \\
\text { (HRt/10 years) }\end{array}$ & 3220 & 18461 & 0.98 (0.95 to 1.02$)$ & 4496 & 17647 & 0.97 (0.91 to 1.02 ) & 1655 & 18540 & 0.87 (0.77 to 0.98 ) \\
\hline \multicolumn{10}{|l|}{ Cumulative exposure } \\
\hline 0 unit-years & 2931 & 17726 & 1 & 2106 & 8434 & 1 & 825 & 9293 & 1 \\
\hline $0.5-9$ unit-years & 839 & 6626 & 1.07 (0.92 to 1.24$)$ & 348 & 1432 & 0.97 (0.76 to 1.24$)$ & 491 & 5193 & 1.09 (0.90 to 1.31$)$ \\
\hline 9.5-28 unit-years & 959 & 5864 & 1.04 (0.91 to 1.19$)$ & 682 & 2484 & 1.11 (0.93 to 1.33$)$ & 277 & 3380 & 0.86 (0.71 to 1.04$)$ \\
\hline 28.5-208 unit-years & 1422 & 5972 & 0.99 (0.87 to 1.14$)$ & 1360 & 5298 & $1.00(0.87$ to 1.16$)$ & 62 & 674 & 0.91 (0.63 to 1.31$)$ \\
\hline Test for trend $\ddagger$ & & & $p=0.27$ & & & $p=0.20$ & & & $p=0.09$ \\
\hline $\begin{array}{l}\text { Test for trend among } \\
\text { exposed only§ }\end{array}$ & & & $\mathrm{p}=0.26$ & & & $p=0.15$ & & & $p=0.07$ \\
\hline
\end{tabular}


Table 5 Cardiovascular disease mortality and cumulative extremely low-frequency magnetic field exposure

\begin{tabular}{|c|c|c|c|c|c|c|}
\hline & \multicolumn{6}{|c|}{ Cumulative exposure (tertiles among ever low and high exposed) } \\
\hline & 0 unit-years & $0.5-9$ unit-years & 9.5-28 unit-years & 28.5-208 unit-years & Test for trend* & $\begin{array}{l}\text { Test for trend amongst } \\
\text { low or high exposedt }\end{array}$ \\
\hline Person-years in subcohort & 17726 & 6626 & 5864 & 5972 & & \\
\hline \multicolumn{7}{|c|}{ Ischaemic heart diseases (IHD) } \\
\hline Cases & 1589 & 402 & 501 & 800 & & \\
\hline $\mathrm{HR} \ddagger(95 \% \mathrm{Cl})$ & 1 & 0.98 (0.82 to 1.17$)$ & 0.98 (0.84 to 1.14$)$ & $0.98(0.85$ to 1.14$)$ & 0.21 & 0.18 \\
\hline \multicolumn{7}{|c|}{ Acute myocardial infarction (AMI) } \\
\hline Cases & 1078 & 289 & 367 & 547 & & \\
\hline $\mathrm{HR} \ddagger(95 \% \mathrm{Cl})$ & 1 & 1.01 (0.84 to 1.22$)$ & 1.05 (0.89 to 1.24$)$ & $1.02(0.87$ to 1.19$)$ & 0.18 & 0.17 \\
\hline \multicolumn{7}{|l|}{ Subacute and chronic IHD } \\
\hline Cases & 511 & 113 & 135 & 256 & & \\
\hline $\mathrm{HR} \ddagger(95 \% \mathrm{Cl})$ & & 0.92 (0.71 to 1.19 ) & 0.83 (0.66 to 1.04 ) & 0.93 (0.76 to 1.12$)$ & 0.41 & 0.35 \\
\hline \multicolumn{7}{|l|}{ Other cardiac causes } \\
\hline Cases & 525 & 146 & 176 & 237 & & \\
\hline $\mathrm{HR} \ddagger(95 \% \mathrm{Cl})$ & 1 & 1.00 (0.79 to 1.26 ) & 1.03 (0.84 to 1.27 ) & 0.97 (0.80 to 1.19$)$ & 0.86 & 0.84 \\
\hline \multicolumn{7}{|l|}{ Arrhythmias } \\
\hline Cases & 67 & 24 & 26 & 30 & & \\
\hline $\mathrm{HR} \ddagger(95 \% \mathrm{Cl})$ & 1 & 1.14 (0.67 to 1.95$)$ & 1.32 (0.83 to 2.09 ) & 0.99 (0.61 to 1.61$)$ & 0.64 & 0.55 \\
\hline \multicolumn{7}{|l|}{ Atherosclerosis } \\
\hline Cases & 54 & 18 & 17 & 32 & & \\
\hline $\mathrm{HR} \ddagger(95 \% \mathrm{Cl})$ & 1 & 1.61 (0.88 to 2.95 ) & 1.10 (0.62 to 1.95$)$ & 1.03 (0.64 to 1.67$)$ & 0.35 & 0.38 \\
\hline \multicolumn{7}{|l|}{ Cerebrovascular diseases } \\
\hline Cases & 541 & 190 & 179 & 233 & & \\
\hline $\mathrm{HR} \ddagger(95 \% \mathrm{Cl})$ & 1 & 1.19 (0.96 to 1.48$)$ & 1.12 (0.92 to 1.36$)$ & $0.98(0.80$ to 1.19$)$ & 0.45 & 0.49 \\
\hline
\end{tabular}

of the subtypes (AMI: third tertile of exposed vs background HR 1.02 (95\% CI 0.87 to 1.19); arrhythmias: third tertile of exposed vs background HR 0.75 (95\% CI 0.44 to 1.29 )). To test alternate definitions of exposure, first, the sex-stratified analyses were performed using sex-specific cut points in the analyses of cumulative exposure and CVD risk. Second, analyses were restricted to subjects who ever had a paying job. Third, analyses were performed on the entire cohort, substituting an exposure level of 0 for jobs that could not be coded. Fourth, the analyses were repeated without the imputation of job duration. Last, different lag-times of 10, 15 and 20 years were implemented, ensuring an equal effective lag-time across the cases and subcohort. These sensitivity analyses did not change the results, and resulted in only slightly different HRs. Ever low or high exposed versus background exposure for CVD changed from a HR of 1.02 to values between 1.01 and 1.06, for AMI from 1.03 to values from 1.01 to 1.06 and for arrhythmias from 1.11 to values ranging from 1.04 to 1.16 . Similarly, in the categorical analysis of cumulative exposure, HRs for the third tertile of exposed category versus background exposure changed from 0.99 to values between 0.97 and 1.02 for CVD, from 1.01 to values ranging from 0.97 to 1.05 for AMI, and from 0.99 to values ranging from 0.83 to 1.08 for arrhythmias. None of the analyses became statistically significant in the sensitivity analyses.

\section{DISCUSSION}

This study examined the association between occupational extremely low-frequency magnetic field (ELF-MF) exposure and risk of mortality due to CVD in a general population cohort.
Occupational ELF-MF exposure appeared not to be associated with total CVD mortality or any of its cause-specific subtypes.

The link between ELF-MF exposure and CVD outcomes was originally put forward on the basis of double-blind experiments showing a reversible relationship between ELF-MF exposure and reduced HRV in humans. ${ }^{2}$ Changes in HRV have, in turn, been shown to be related to cardiac autonomic activity, and to be a useful indicator in identifying patients with a higher risk of CVD morbidity and mortality. ${ }^{19}$ The initial findings of Savitz et $\mathrm{al}^{4}$ of an increase in risk of AMI and arrhythmia-related mortality due to occupational ELF-MF exposure in a population of utility workers seemed to be in line with this proposed mechanism. However, subsequent experimental and epidemiological studies failed to replicate the relationship between ELF-MF exposure and reduced HRV and associated CVD risk. ${ }^{5-11} 1320$ This led Kheifets et $a l^{12}$ to conclude in their literature review that the current evidence does not support a relationship between occupational exposures to electric and magnetic fields and CVD.

This present study tried to improve on earlier epidemiological studies in several ways. First, because of the size of the cohort, the number of cases used in the analyses is higher than in many previous epidemiological studies on occupational ELF-MF exposure and CVD mortality (see Kheifets et $a l^{12}$ for an overview). Second, in this study, it was possible to look at the possible sex-specific effects of ELF-MF. Often women are under-represented in industry-based studies prohibiting the study of health effects by sex. Third, because of the detailed questionnaire data obtained at baseline, it was possible to consider and adjust for many potentially confounding lifestyle factors. This has been a limitation in earlier epidemiological studies, where such detailed information 
was not always available. ${ }^{413}$ Fourth, occupational histories were available for $75 \%$ of the cases and $78 \%$ of the subcohort members up to baseline. Since the average age at baseline was high (64 years for the cases and 62 years for the subcohort, following the age inclusion criterion of 55-69 years), and many subjects had already retired ( $87 \%$ of the cases and $78 \%$ of the subcohort), it was possible to consider multiple exposure metrics that were lifelong for most subjects. In earlier population-based cohort studies, a single occupation was mostly used to determine occupational exposure, potentially missing relevant periods with ELF-MF exposure. 810

There were several limitations in this study. As in other epidemiological studies, the CVD outcomes were assessed through the use of death certificates. These have been suggested to have a high specificity but low sensitivity, ${ }^{21} 22$ indicating that death certificates are likely to underestimate the number of cardiovascular causes. ${ }^{22}$ However, this misclassification is likely to be non-differential with respect to the exposure classification to ELF-MF and, therefore, would not bias the results, yet would limit the power of this study to detect an effect. The potential loss of power in this study is being offset by the large size of the cohort.

The cohort consists of an elderly, mostly retired population, which limited the ability to look at effects of very recent and concurrent exposure. Furthermore, occupational histories were only available till baseline, meaning that there was no occupational information for the follow-up period (10 years). This may have led to an underestimate of the occupational ELF-MF exposure in the younger part of the population that has not yet retired $(13 \%$ of the cases and $22 \%$ of the subcohort). Since these people would also have a lower risk of CVD mortality, this might bias the results. This possible bias was investigated in two ways. First, a stratified analysis was performed by those who were retired at baseline, versus those who were still working at baseline. Second, additional analyses were performed in which the exposure was lagged 10,15 and 20 years before the end of follow-up. These analyses did not lead to different conclusions (results not shown).

Last, although there are a high number of cases in the cohort, the number of (high) exposed cases may be lower as compared with industry-based cohorts. Also, the exposure assessment in industry-based cohorts is often more detailed, using measurements to implement an exposure classification for the jobs within a certain industry. 411

This study used a modified ELF-JEM based on a JEM developed by Bowman et al. ${ }^{18}$ In the modified JEM, subjects are grouped semiquantitatively in background, low and high exposure categories, rather than using original quantitative estimates. This modification was necessary since the original JEM was only intensity-based and did not necessarily reflect the probability of exposure higher than background. The modified ELF-JEM takes both the intensity and estimated probability of exposure into account, but, therefore, necessarily became semiquantitative as quantitative estimates of probability of exposure were not available. We performed additional epidemiological analyses using the original ELF-JEM, and the results did not change.

In conclusion, this study does not provide support for the hypothesis that occupational exposure to ELF-MF increases the risk of CVD mortality.
Contributors All authors have been involved with the work submitted, share responsibility for and approved the submission of the manuscript. None of the authors declare a conflict of interest.

Funding This work was supported by The Netherlands Organization for Health Research (ZonMW) within the programme Electromagnetic Fields and Health Research, under grant numbers 85200001 and 85800001 .

\section{Competing interests None.}

Patient consent Obtained.

Ethics approval Institutional review boards of The Netherlands Organization for Applied Scientific Research TNO (Zeist) and Maastricht University (Maastricht).

Provenance and peer review Not commissioned; externally peer reviewed.

\section{REFERENCES}

1 Milham S Jr. Mortality in workers exposed to electromagnetic fields. Environ Health Perspect 1985;62:297-300.

2 Sastre A, Cook MR, Graham C. Nocturnal exposure to intermittent $60 \mathrm{~Hz}$ magnetic fields alters human cardiac rhythm. Bioelectromagnetics 1998;19:98-106.

3 Sastre A, Graham C, Cook MR. Brain frequency magnetic fields alter cardiac autonomic control mechanisms. Clin Neurophysiol 2000;111:1942-8.

4 Savitz DA, Liao D, Sastre A, et al. Magnetic field exposure and cardiovascular disease mortality among electric utility workers. Am J Epidemiol 1999;149:135-42.

5 Sahl J, Mezei G, Kavet $\mathrm{R}$, et al. Occupational magnetic field exposure and cardiovascular mortality in a cohort of electric utility workers. Am J Epidemiol 2002:156:913-18.

6 Ahlbom A, Feychting M, Gustavsson A, et al. Occupational magnetic field exposure and myocardial infarction incidence. Epidemiology 2004;15:403-8.

7 Cooper AR, Van Wijngaarden E, Fisher SG, et al. A population-based cohort study of occupational exposure to magnetic fields and cardiovascular disease mortality. Ann Epidemiol 2009;19:42-8.

8 Hakansson N, Gustavsson P, Sastre A, et al. Occupational exposure to extremely low frequency magnetic fields and mortality from cardiovascular disease. Am J Epidemiol 2003;158:534-42.

9 Johansen $C$, Feychting M, Moller $M$, et al. Risk of severe cardiac arrhythmia in male utility workers: a nationwide danish cohort study. Am J Epidemiol 2002;156:857-61.

10 Mezei G, Cher D, Kelsh M, et al. Occupational magnetic field exposure, cardiovascular disease mortality, and potential confounding by smoking. Ann Epidemiol 2005;15:622-9.

11 Roosli M, Egger M, Pfluger D, et al. Cardiovascular mortality and exposure to extremely low frequency magnetic fields: a cohort study of Swiss railway workers. Environ Health 2008;7:35.

12 Kheifets L, Ahlbom A, Johansen C, et al. Extremely low-frequency magnetic fields and heart disease. Scand J Work Environ Health 2007;33:5-12.

13 Sorahan T, Nichols L. Mortality from cardiovascular disease in relation to magnetic field exposure: findings from a study of UK electricity generation and transmission workers, 1973-1997. Am J Ind Med 2004:45:93-102.

14 van den Brandt PA, Goldbohm RA, van 't Veer P, et al. A large-scale prospective cohort study on diet and cancer in The Netherlands. J Clin Epidemiol 1990:43:285-95.

15 CBS. Beroepsclassificatie 1984. Voorburg/Heerlen: CBS, 1984.

16 Koeman T, S M Offermans N, Christopher-de Vries Y, et al. JEMs and incompatible occupational coding systems: effect of manual and automatic recoding of job codes on exposure assignment. Ann Occup Hyg 2012 Jul 17. [Epub ahead of print].

17 Huss A, Vermeulen R, Bowman J, et al. Electric shocks at work in Europe: development of a job exposure matrix. Occup Environ Med 2012Nov 22. [Epub ahead of print].

18 Bowman JD, Touchstone JA, Yost MG. A population-based job exposure matrix for power-frequency magnetic fields. J Occup Environ Hyg 2007;4:715-28.

19 Lombardi F, Stein PK. Origin of heart rate variability and turbulence: an appraisal of autonomic modulation of cardiovascular function. Front Physiol 2011;2:95.

20 Johansen C, Olsen JH. Mortality from amyotrophic lateral sclerosis, other chronic disorders, and electric shocks among utility workers. Am J Epidemiol 1998; 148:362-8

21 Sington JD, Cottrell BJ. Analysis of the sensitivity of death certificates in 440 hospital deaths: a comparison with necropsy findings. J Clin Pathol 2002:55:499-502.

22 Harriss $L R$, Ajani $A E$, Hunt $D$, et al. Accuracy of national mortality codes in identifying adjudicated cardiovascular deaths. Aust N Z J Public Health 2011;35:466-76. 